\title{
Outcomes of therapeutic keratoplasty for severe infectious keratitis in Chongqing, a 16-year experience
}

This article was published in the following Dove Press journal: Infection and Drug Resistance

\author{
Qi Zhang' \\ Min Zhao' \\ Mei $X u^{\prime}$ \\ Fengjuan $\mathrm{Gu}^{\prime}$ \\ Quan Liu' \\ Yuan Chen ${ }^{2}$ \\ Haiqi Zhang' \\ Aize Kijlstra ${ }^{3}$
}

'The First Affiliated Hospital of Chongqing Medical University, Department of Ophthalmology, Chongqing Key Laboratory of Ophthalmology and Chongqing Eye Institute, Chongqing, People's Republic of China; ${ }^{2}$ Chongqing Vision Institute, Chongqing, People's Republic of China; ${ }^{3}$ University Eye Clinic Maastricht, Maastricht, the Netherlands

Correspondence: Min Zhao

The First Affiliated Hospital of Chongqing Medical University, Department of

Ophthalmology, I YouYi Road, YuZhong

District, Chongqing 400016, People's

Republic of China

Tel +86238901 3169

Email eyedoctorz@I26.com
Purpose: This study aimed to evaluate the outcomes of therapeutic keratoplasty for severe infectious keratitis in Chongqing (Southwest China).

Patients and methods: The records of 561 eyes that underwent therapeutic keratoplasty for refractory microbial keratitis from 2001 to 2016 were analyzed in this retrospective study. Data included demographic information, microbiological investigations, associated factors, graft size, preoperative status, postoperative complications, and final anatomical outcomes.

Results: Trauma was the most common cause $(267,47.6 \%)$ for corneal ulcers leading to therapeutic keratoplasty. The etiological diagnosis included bacterial keratitis ( 80 eyes, $14.3 \%$ ), fungal keratitis (317 eyes, 56.5\%), acanthamoeba keratitis ( 3 eyes, $0.5 \%$ ), and mixed bacteria/fungal infection (15 eyes, 2.7\%). Anatomical success was achieved for 492 eyes $(87.7 \%)$, with bacterial keratitis having a better outcome than fungal and mixed infections. Diabetes and preoperative time $\geq 30$ days were significantly associated with anatomical failure in the multivariate logistic regression $(P=0.028$ and $P=0.022$, respectively). Patients with hypopyon, corneal perforation, surgical delay, and/or large graft size had a higher incidence of postoperative complications (reinfection, cataract, glaucoma, hyphema, or graft rejection) $(P<0.05)$.

Conclusion: Therapeutic keratoplasty was an effective procedure in managing refractory infectious keratitis. Prompt and appropriate surgery would result in fewer complications and better outcomes.

Keywords: therapeutic keratoplasty, infectious keratitis, corneal ulcer

\section{Introduction}

Infectious keratitis is an important cause of visual impairment and blindness worldwide, especially in developing countries. ${ }^{1}$ Without prompt and appropriate treatment, the severe infection may result in wide range of corneal infiltration, extensive anterior chamber reaction, perforation, and endophthalmitis possibly leading to enucleation. ${ }^{1-4}$ The presentation and outcomes of infectious keratitis may depend on different region and epidemiology, thus necessitating studies to be performed throughout the world. ${ }^{5}$ An analysis of the management for severe infectious keratitis in Southwest China has not yet been reported on a large scale, that was one of the reasons for performing this study. Therapeutic keratoplasty (TKP) for severe infectious keratitis accounts about $29.9 \%$ of all grafts operated at our ophthalmology center for cornea transplantations in Chongqing, with the patient population from Southwest China. The primary goal of TKP is to save the eyeball 
integrity, whereby visual rehabilitation is a secondary consideration. ${ }^{6}$ Infection recurrence and high incidence of complications after TKP are still the significant challenges for oculists. Investigation of risk factors could be helpful in preventing the severe infection recurrence. Moreover, analyzing the clinical characteristics, postoperative complications, and the association would be beneficial in improving the outcomes. Furtherly, evaluating outcomes would be good in improving treatment processes and protocols for severe infectious keratitis.

\section{Materials and methods}

The records of all TKP procedures performed during a 16year period (from January 2001 to December 2016) at the First Affiliated Hospital of Chongqing Medical University (Chongqing, China) were retrospectively reviewed. Data included demographic parameters, causative agents, preoperative time (duration from the infection symptom onset to surgery), donor graft size, postoperative complications and outcomes. The etiological diagnosis was made based on the clinical performance combined with laboratory tests, including corneal scraping, smear, culture, and pathological examination (excised corneal tissue sent for pathological microscopy and microbial culture) in all cases, while confocal microscopy was used in some cases. Medical history and reaction to antimicrobials were also considered for diagnosis. Clinical parameters included anatomical status, graft status, intraocular pressure, anterior segment inflammation, and postoperative complications. Patients who did not attend follow-up for at least 3 months were excluded. Outcome evaluation was done after 3 months. All cases were unilateral. The subjects provided their written informed consent to participate in this study. Procedures of this study were conformed the ethical guidelines of the Declaration of Helsinki. The study protocols were reviewed and approved by the Ethics Committee in the First Affiliated Hospital of Chongqing Medical University (No. 2014-76).

\section{Treatment before surgery}

During presentation, scrapings of the cornea were sent out for Gram staining and microbial culture (blood agar and Sabouraud dextrose agar for bacterial and fungal cultures) including an antibiotic susceptibility test. Some eyes were checked with confocal microscopy to search hyphae or acanthamoeba cysts. Antimicrobial therapy was given depending on the laboratory test, confocal microscopy evaluation, and clinical features, and included topical and/or systemic antibiotics or antifungals, which were continued until the time of surgery. TKP was performed if the infiltrates penetrated into the deeper stroma and/or affected the limbus even up to the sclera, and in cases with impending perforation, perforation, or recurrent hypopyon. A B-scan ultrasound was performed to exclude endophthalmitis before the operation.

\section{Surgical procedure}

Five ophthalmologists performed the operations. Donor corneas from the Chongqing eye bank were used. The resection site was chosen to be larger than the whole infiltrate area. The donor graft diameter was $0.5-0.75 \mathrm{~mm}$ larger than the host bed for penetrating keratoplasty (PKP) or $0.25 \mathrm{~mm}$ larger for lamellar keratoplasty (LKP). The excised host tissue was sent out for microbiological and pathological investigation. The selection of PKP or LKP was made for each eye depending on the depth of infiltration of the cornea. The purulent debris and inflammatory membranes were irrigated and mechanically removed from the anterior chamber. Anterior synechiae was separated as thoroughly as possible. Additional surgical procedures were performed as indicated, including peripheral iridectomy to prevent pupillary block glaucoma, cataract surgery if the infection extended to the lens, and/or anterior vitrectomy. A total of 16 interrupted 10-0 nylon sutures were used for corneal transplantation.

\section{Postoperative treatment}

After surgery, prolongation of antimicrobial treatment depended on the severity of infection and species of etiology. Patients with bacterial keratitis were prescribed topical and systemic antibiotics, in addition to topical steroids and nonsteroidal anti-inflammatory drops. Fungal keratitis required longer therapy compared with bacterial keratitis. Patients with fungal keratitis received oral itraconazole for 3 weeks. Low-concentration topical steroids were given after 4 weeks from TKP if the fungal infection was controlled without recurrence. All topical antifungal agents were tapered and discontinued about 60 days after TKP. Patients with acanthamoeba keratitis were treated with topical chlorhexidine, fluconazole, and antibiotics eye drops. Patients with undetermined pathogen were prescribed broad-spectrum antimicrobial agents (tobramycin, levofloxacin, or gatifloxacin, and so on). Atropine ointment was used in all cases with hypopyon. Follow-up was done weekly for 1 month, monthly for 3 months, and 3- to 6-monthly thereafter. 


\section{Statistical analysis}

Statistical analysis was performed using SPSS software version 16.0 (SPSS Inc. IL, USA). For description, mean \pm standard deviation (SD) was expressed for quantitative variables, and frequency (\%) was expressed for categorical data. The chi-square test and Fisher's exact test were performed for categorical variables. Univariable and multivariable analysis was conducted using the logistic regression model. A $P$-value less than 0.05 was considered significant. Associations were evaluated using odds ratio (OR) with $95 \%$ confidence interval (CI).

\section{Results}

In the reviewed 16-year period, 598 patients underwent TKP for severe infectious keratitis (usually deemed as hopeless cases and advised evisceration) and 37 patients were lost to follow-up. Thus, 561 patients (561 eyes) were analyzed. The average age of the patients was 53.3 \pm 15.4 years (range $3-88$ years), and 355 (63.3\%) were male. Overall, $455(455 / 561,81.1 \%)$ patients were from rural districts in Southwest China. The average preoperative time (duration from the infection symptom onset to surgery) was $31 \pm 19.3$ days (range 7-68 days). The average followup duration was $21 \pm 13.5$ months (range 3-38 months). PKP was done in 454 patients $(454 / 561,80.9 \%)$, and LKP was performed in 107 patients $(107 / 561,19.1 \%)$.

\section{Characteristics of keratitis}

Of the 561 patients, the dominating associated factor was trauma (267 patients, $47.6 \%$ ), especially the plant-induced trauma (165 patients, 29.4\%). Poorly controlled diabetes (fasting blood glucose $>7 \mathrm{mmol} / \mathrm{L}$ ) was present in 70 patients $(12.5 \%)$. Other patients had underlying diseases, such as $47(8.4 \%)$ with ocular surface disorders, 24 (4.3\%) with upper respiratory infection, and 21 (3.7\%) with dacryocystitis. Moreover, 19 patients (3.4\%) wore contact lenses before infectious keratitis. In 96 patients (17.1\%), no specific associated factors could be established (Table 1).

\section{Microbiological diagnosis}

Of 561 patients, the microbiological diagnosis was bacterial keratitis in 80 patients (14.3\%), fungal keratitis in 317 patients $(56.5 \%)$, and mixed fungal and bacterial infection in 15 patients $(2.7 \%)$. Three eyes $(0.5 \%)$ were diagnosed with acanthamoeba keratitis. In 146 patients (26.0\%), no species or etiology could be identified. In bacterial keratitis, the culture-positive cases were 43 (43/95, 45.3\%); of these, the commonest species was the coagulase-negative Staphylococcus (20/43, 46.5\%), followed by Pseudomonas (16/43, 37.2\%). In fungal keratitis, the culture-positive cases were 218 (218/332, 65.7\%); of these, the commonest species was Fusarium (101/218, 46.3\%), followed by Aspergillus (49/218, 22.5\%). As plant-induced traumas showed the highest incidence (165/561, 29.4\%), fungal infections (mixed and pure fungal) were more frequent $(134 / 165,81.2 \%)$ in this subgroup compared with bacterial infections $(22 / 165,13.3 \%)$ (Table 1).

\section{Therapeutic outcomes}

A total of 492 patients $(492 / 561,87.7 \%)$ achieved an anatomical success with the reestablishment of ocular integrity. A higher success rate was obtained in patients with a bacterial infection $(76 / 80,95.0 \%)$ compared with those with a fungal $(267 / 317,84.2 \%, P=0.012)$ or mixed bacterial and fungal infection $(11 / 15,73.3 \%, P=0.020)$ (Table 2).

Logistic regression was performed to analyze whether the anatomical failure was affected by the factors shown in Table 3. Diabetes and preoperative time (duration from symptom onset to surgery) $\geq 30$ days were found to be significantly associated with poor outcome using both univariate ( $P=0.039$ and $P=0.011$, respectively) and multivariate $(P=0.028$ and $P=0.022$, respectively) analyses.

\section{Postoperative complications}

In the postoperative period, reinfection occurred in 81 grafts $(81 / 561,14.4 \%)$; a repeat TKP was performed in 23 patients. Ultimately $69(69 / 561,12.3 \%)$ eyes progressed to severe and uncontrolled infections that required evisceration or enucleation. The associated factors for reinfection were recurrent hypopyon, corneal perforation, and preoperative time ( $\geq 30$ days) $(P=0.036, P=0.028$, and $P=0.0003$, respectively). Hypopyon, corneal perforation, preoperative time, and large graft size $(\geq 8.75 \mathrm{~mm})$ all showed a significant association with cataract $(P=0.007$, $P=0.002, P=0.006$, and $P=0.019$, respectively) and secondary glaucoma $(P=0.011, P=0.007, P=0.015$, and $P=0.027$, respectively). Preoperative time and large graft size were both associated with hyphema $(P=0.010$ and $P=0.043$, respectively). Large graft size was significantly associated with graft rejection $(P=0.015)$ (Table 4$)$.

\section{Discussion}

This retrospective study reviewed a 16-year period and represented a patient population from Southwest China, a 
Table I Etiological diagnosis and associated factors for infectious keratitis

\begin{tabular}{|c|c|c|c|c|c|c|}
\hline \multirow[t]{2}{*}{ Associated factors } & \multirow[t]{2}{*}{ Bacteria } & \multirow[t]{2}{*}{ Fungus } & \multicolumn{2}{|l|}{ Etiology (No.) } & \multirow[t]{2}{*}{ Unproven } & \multirow[t]{2}{*}{ Total (\%) } \\
\hline & & & Acanthamoeba & Mixed & & \\
\hline Plant-induced trauma & 16 & 128 & 0 & 6 & 15 & $165(29.4)$ \\
\hline Other trauma & 23 & 36 & 1 & 4 & 38 & $102(18.2)$ \\
\hline Diabetes & 10 & 33 & 0 & I & 26 & $70(12.5)$ \\
\hline Ocular surface disorder & 7 & 28 & 0 & 0 & 12 & $47(8.4)$ \\
\hline Respiratory infection & 3 & 17 & 0 & 0 & 4 & $24(4.3)$ \\
\hline Dacryocystitis & 9 & 0 & 0 & 0 & 12 & $21(3.7)$ \\
\hline Contact lens & 8 & 0 & 2 & 0 & 9 & $19(3.4)$ \\
\hline Others & 2 & 6 & 0 & 0 & 9 & $17(3.0)$ \\
\hline None & 2 & 69 & 0 & 4 & 21 & $96(17.1)$ \\
\hline Total (\%) & $80(14.3 \%)$ & $317(56.5 \%)$ & $3(0.5 \%)$ & I5 (2.7\%) & $146(26.0 \%)$ & $561(100 \%)$ \\
\hline
\end{tabular}

Abbreviation: Mixed, mixed bacterial and fungal infection.

Table 2 Anatomical outcomes after therapeutic keratoplasty

\begin{tabular}{|l|l|}
\hline Etiology (No.) & Anatomical Success (\%) \\
\hline Bacteria (80) & $76(95.0 \%)$ \\
Fungus (3I7) & $267(84.2 \%)$ \\
Acanthamoeba (3) & $2(66.7 \%)$ \\
Mixed (15) & $11(73.3 \%)$ \\
Unproven (146) & $136(93.2 \%)$ \\
Total (56I) & $492(87.7 \%)$ \\
\hline
\end{tabular}

Notes: $P$-value for anatomical success for bacterial and fungal keratitis is 0.012 ; $P$ value for anatomical success for bacterial and mixed keratitis is 0.020 ; $P$-value for anatomical success for fungal and mixed keratitis is 0.28

Abbreviation: Mixed, mixed bacterial and fungal infection.

region with typical climate, geography, and ethnic population. In this study, the majority of the patients $(455 / 561$, $81.1 \%$ ) were farmers or came from rural areas, and the dominant associated factor was trauma (267/561, 47.6\%), especially plant-induced trauma $(165 / 561,29.4 \%)$, often leading to fungal keratitis $(332 / 561,59.2 \%)$. The results were in agreement with some studies in Asian developing regions and countries, where trauma leading to fungal keratitis was often observed. ${ }^{7-9}$ On the contrary, in Asian developed regions, such as Beijing, Shanghai, Hong Kong, and Japan, the epidemiology of infectious keratitis resembled that seen in North American and European regions. Bacteria keratitis was the main category, and wearing contact lenses, which was rarely encountered in our study, was the predisposing factor. ${ }^{5,10-12}$ Geographical variation may lead to a different epidemiology of infectious keratitis. ${ }^{13-17}$ For instance, Fusarium was the commonest fungus in our study, as in Shandong and Singapore, while Aspergillus was the major fungal species causing keratitis in Taiwan, North India, and Nepal. In this study, coagulase-negative Staphylococcus was the commonest cause for bacterial keratitis, as in North India and Nepal, while it was Pseudomonas in some other studies. It was speculated that the present study might be biased with cases caused by Staphylococcus because the condition of patients infected with Pseudomonas might already be too severe to allow their transfer to our center and already had undergone enucleation at their local hospitals.

A large number of culture-negative cases were reported in this study, similar to previous reports, which made accurate medication troublesome. ${ }^{3,16}$ The false culture-negative suppurative keratitis cases could be explained by the fact that before coming to the institute, most patients already showed long disease duration and had been treated at local medical facilities. No infecting organism could be identified in more than one fourth of the patients $(146 / 561,26.0 \%)$. The data in this study prompted that rural patients served by a local ophthalmologist should be timely referred to a corneal specialist depending upon the presentation.

In the present study, anatomical restoration was achieved for the majority of patients $(492 / 561,87.7 \%)$, which was consistent with previous reports. ${ }^{3,16,18}$ The anatomical success rate was higher in TKP performed for bacterial keratitis than for fungal ulcers or mixed fungal and bacterial infection, which might be due to the high morbidity of fungal recurrences. The criteria for donor tissue quality were not as strict as for optical PKP because the primary goal of TKP was to remove the refractory corneal infection and reestablish eyeball integrity. ${ }^{19}$ In our study cryopreserved corneas were used as emergency keratoplasty for severe keratitis with perforation or that affected the limbus and sclera when no fresh donor cornea was available. Other reports also demonstrated that cryopreserved donor corneas could be effectively 
Table 3 Logistic regression of characteristics related to anatomical failure after therapeutic keratoplasty

\begin{tabular}{|c|c|c|c|c|c|c|}
\hline \multirow[t]{2}{*}{ Characteristics } & \multirow{2}{*}{$\begin{array}{l}\begin{array}{l}\text { Anatomical } \\
\text { Failure }\end{array} \\
\mathrm{N}=69\end{array}$} & \multirow{2}{*}{$\begin{array}{l}\text { Anatomical Success } \\
\mathrm{N}=492\end{array}$} & \multicolumn{2}{|c|}{ Univariable analysis } & \multicolumn{2}{|c|}{ Multivariable analysis * } \\
\hline & & & $P$-value & OR (95\% Cl) & $P$-value & OR $(95 \% \mathrm{Cl})$ \\
\hline Age & $54.1 \pm 16.3$ & $52.9 \pm 17.8$ & 0.509 & $0.176(0.727-1.901)$ & 0.570 & $1.361(0.470-3.944)$ \\
\hline Male gender & $4 \mathrm{l}(59.4 \%)$ & $314(63.8 \%)$ & 0.478 & $0.830(0.496-1.389)$ & 0.663 & $0.782(0.258-2.367)$ \\
\hline Trauma & 39 (56.5\%) & $228(46.3 \%)$ & 0.115 & $1.505(0.706-2.50 \mathrm{I})$ & 0.716 & $1.366(0.254-7.343)$ \\
\hline Diabetes & $14(20.3 \%)$ & 56 (II.4\%) & 0.039 & $1.982(1.035-3.794)$ & 0.028 & $2.796(1.115-7.009)$ \\
\hline Ocular surface disorder & 9 (13.0\%) & 38 (7.7\%) & 0.140 & $1.792(0.826-3.889)$ & 0.328 & $2.200(0.454-10.662)$ \\
\hline Respiratory infection & $5(7.2 \%)$ & $19(3.9 \%)$ & 0.201 & $1.945(0.702-5.389)$ & 0.695 & $1.790(0.097-32.946)$ \\
\hline Dacryocystitis & $2(2.9 \%)$ & $19(3.9 \%)$ & 0.694 & $0.743(0.169-3.262)$ & 0.929 & $0.867(0.037-20.103)$ \\
\hline Contact lens & $2(2.9 \%)$ & 17 (3.5\%) & 0.811 & $0.834(0.188-3.691)$ & 0.938 & $0.901(0.065-12.544)$ \\
\hline Hypopyon & $54(78.3 \%)$ & 341 (69.3\%) & 0.130 & $1.594(0.872-2.914)$ & 0.208 & $1.828(0.7|5-4.67|)$ \\
\hline Corneal perforation & $15(21.7 \%)$ & 71 (I4.4\%) & 0.118 & I.647 (0.882-3.077) & 0.064 & $1.839(0.965-3.502)$ \\
\hline Preoperative time ( $\geq 30$ days) & $34(49.3 \%)$ & 165 (33.5\%) & 0.011 & $1.925(1.159-3.199)$ & 0.022 & $2.472(1.143-5.347)$ \\
\hline Size of graft $(\geq 8.75 \mathrm{~mm})$ & $53(76.8 \%)$ & $336(68.3 \%)$ & 0.153 & $1.538(0.852-2.776)$ & 0.122 & $2.025(0.828-4.949)$ \\
\hline
\end{tabular}

Note: *All variables in the univariable analysis were included in the multivariable analysis.

Abbreviations: OR, odds ratio; $95 \% \mathrm{Cl}, 95 \%$ confidence interval.

applied for TKP even in patients with a large corneal perforation. ${ }^{20,21}$ The use of cryopreserved donor corneas was also necessary in view of the extreme shortage of donor corneas in China. ${ }^{19}$
The postoperative complications also affected the restoration of visual acuity. In the present study, reinfection, cataract, and secondary glaucoma were all more common in patients with recurrent hypopyon, corneal perforation,

Table 4 Postoperative complications and associated characteristics

\begin{tabular}{|c|c|c|c|c|c|}
\hline \multirow[t]{2}{*}{ Characteristic } & \multirow[t]{2}{*}{ Complications } & \multirow{2}{*}{$\begin{array}{l}\text { Present } \\
\text { No.of patients (\%) }\end{array}$} & \multirow{2}{*}{$\begin{array}{l}\text { Absent } \\
\text { No.of patients (\%) }\end{array}$} & \multirow[t]{2}{*}{ OR $(95 \% \mathrm{Cl}) *$} & \multirow[t]{2}{*}{$P$-value * } \\
\hline & & & & & \\
\hline \multirow[t]{5}{*}{ Hypopyon } & Reinfection & $65 / 395(16.5)$ & $16 / 166(9.6)$ & $1.847(1.034-3.298)$ & 0.036 \\
\hline & Rejection & 124/395 (31.4) & $43 / 166$ (25.9) & $1.309(0.87 \mid-1.966)$ & 0.194 \\
\hline & Cataract & $303 / 395(76.7)$ & $109 / 166(65.7)$ & $1.722(1.159-2.560)$ & 0.007 \\
\hline & Glaucoma & $117 / 395(29.6)$ & $32 / 166(19.3)$ & $1.762(1.133-2.742)$ & 0.011 \\
\hline & Hyphema & $91 / 395(23.0)$ & $30 / 166(18.1)$ & $1.357(0.857-2.148)$ & 0.192 \\
\hline \multirow[t]{5}{*}{ Corneal perforation } & Reinfection & $19 / 86(22.1)$ & $62 / 475(13.1)$ & $1.889(1.063-3.357)$ & 0.028 \\
\hline & Rejection & $31 / 86(36.0)$ & I36/475 (28.6) & $1.405(0.867-2.278)$ & 0.166 \\
\hline & Cataract & 75/86 (87.2) & $337 / 475$ (70.9) & $2.792(1.439-5.418)$ & 0.002 \\
\hline & Glaucoma & $33 / 86(38.4)$ & I I6/475 (24.4) & $1.927(1.190-3.122)$ & 0.007 \\
\hline & Hyphema & $23 / 86(26.7)$ & $98 / 475(20.6)$ & $1.404(0.830-2.378)$ & 0.205 \\
\hline \multirow{5}{*}{$\begin{array}{l}\text { Preoperative time } \\
\text { ( } \geq 30 \text { days) }\end{array}$} & Reinfection & $43 / 199(21.6)$ & $38 / 362(10.5)$ & $2.350(1.460-3.784)$ & 0.0003 \\
\hline & Rejection & $68 / 199(34.2)$ & $99 / 362(25.3)$ & $1.379(0.950-2.003)$ & 0.091 \\
\hline & Cataract & $160 / 199(80.4)$ & $252 / 362(69.6)$ & 1.791 (1.182-2.713) & 0.006 \\
\hline & Glaucoma & $65 / 199$ (32.7) & $84 / 362(23.2)$ & $1.605(1.094-2.357)$ & 0.015 \\
\hline & Hyphema & $55 / 199(27.6)$ & $66 / 362(18.2)$ & $1.713(1.138-2.580)$ & 0.010 \\
\hline \multirow{5}{*}{$\begin{array}{l}\text { Size of graft } \\
(\geq 8.75 \mathrm{~mm})\end{array}$} & Reinfection & $63 / 389(16.2)$ & $18 / 172(10.5)$ & $1.653(0.947-2.888)$ & 0.075 \\
\hline & Rejection & 128/389 (32.9) & $39 / 172(22.7)$ & $1.672(1.104-2.532)$ & 0.015 \\
\hline & Cataract & $297 / 389(76.3)$ & $115 / 172(66.9)$ & $1.600(1.079-2.374)$ & 0.019 \\
\hline & Glaucoma & II4/389 (29.3) & $35 / 172(20.3)$ & $1.623(1.055-2.495)$ & 0.027 \\
\hline & Hyphema & $93 / 389(23.9)$ & $28 / 172(16.3)$ & $1.616(1.013-2.578)$ & 0.043 \\
\hline
\end{tabular}

Note: *Univariable logistic regression.

Abbreviations: OR, odds ratio; $95 \% \mathrm{Cl}, 95 \%$ confidence interval. 
$\geq 30$ days' course to surgery, and/or large graft size $(P<0.05)$.

The associations were similar to those in previous studies, while preoperative time was rarely reported. These complications might account for the complicated surgery, with extreme difficulty in thoroughly excising infiltrated area, removing purulent membranes, and separating anterior synechiae. ${ }^{22,23}$ Hyphema was associated with surgical delay and the use of large graft, and was often due to the presence of refractory infection and multiple neovascularization. ${ }^{24} \mathrm{~A}$ large graft was a significant associated factor for graft rejection. ${ }^{21}$ In the present study, the diameter of graft was often equal to or larger than $8.75 \mathrm{~mm}(389 / 561,69.3 \%)$, which was due to the extensive range of severe infiltration.

Delayed time to surgery and diabetes were associated with poor outcome with loss of eyeball integrity, as analyzed by multivariable logistic regression. Surgical delay may hamper the prevention of further infection on adjacent tissues, especially toward the limbus and the lens. ${ }^{6}$ Therefore, if an infectious keratitis does not respond to antimicrobial therapy, an early TKP was recommended rather than waiting too long for better outcomes. Untreated diabetes, usually misdiagnosed and ignored, commonly leads to reduced immunity and deterioration of infection, should be given enough attention and active treatment. ${ }^{25}$

This study had some limitations. More than one fourth of the cases remained without an identified etiology. Many cases presented as false culture-negative due to multiple topical and systemic antimicrobial therapies. However, some might have suffered from viral keratitis but could not be identified because of the lack of a routine test method. Another limitation was the short follow-up duration that did not include long-term complications such as graft rejection.

\section{Conclusion}

Overall, the present study suggested that early appropriate diagnosis and antimicrobial therapy were important for treating infectious keratitis. TKP was an effective method, which could be used on an emergent case to rescue the eye from refractory keratitis. It was believed that TKP would achieve less postoperative complications and better clinical outcomes if the operation was performed soon enough to avoid perforation or infiltration expansion.

\section{Acknowledgments}

The authors would like thank all donors enrolled in the present study. They also thank Yetao Luo (Department of Health Statistics, Public Health Department of Chongqing Medical University) for advice on the statistical matters.
The study was supported by the National Key Clinical Specialties Construction Program of China, the National Key R\&D Program of China (2016YFC0904000), the Chongqing Key Laboratory of Ophthalmology (CSTC, 2008CA5003), the National Natural Science Foundation Project (81300754), and the Project of Health Bureau of Chongqing (2016MSXM003). The funding organizations had no role in the design or conduct of this study.

\section{Disclosure}

The authors report no conflicts of interest in this work.

\section{References}

1. Ramamurthy S, Reddy JC, Vaddavalli PK, Ali MH, Garg P. Outcomes of repeat keratoplasty for failed therapeutic keratoplasty. Am J Ophthalmol. 2016;162:83-88 e82. doi:10.1016/j.ajo.2015.11.004

2. Gao H, Song P, Echegaray JJ, et al. Big bubble deep anterior lamellar keratoplasty for management of deep fungal keratitis. $J$ Ophthalmol. 2014;2014:209759. doi:10.1155/2014/209759

3. Sharma N, Jain M, Sehra SV, et al. Outcomes of therapeutic penetrating keratoplasty from a tertiary eye care centre in northern India. Cornea. 2014;33(2):114-118. doi:10.1097/ICO.0000000000000025

4. Shi W, Wang T, Xie L, et al. Risk factors, clinical features, and outcomes of recurrent fungal keratitis after corneal transplantation. Ophthalmology. 2010;117(5):890-896. doi:10.1016/j.ophtha.2009.10.004

5. Ng AL, To KK, Choi CC, et al. Predisposing factors, microbial characteristics, and clinical outcome of microbial keratitis in a tertiary centre in Hong Kong: a 10-year experience. J Ophthalmol. 2015;2015:769436.

6. Jain R, Bhutia KL, Mohan N, Gupta CKC, Ghai A. Outcome of therapeutic keratoplasty in hopeless microbial keratitis cases otherwise advised evisceration. Cornea. 2018;37(2):151-155. doi:10.1097/ICO.0000000000001432

7. Tananuvat N, Punyakhum O, Ausayakhun S, Chaidaroon W. Etiology and clinical outcomes of microbial keratitis at a tertiary eye-care center in northern Thailand. J Med Assoc Thai. 2012;95 (Suppl 4):S8-S17.

8. Tewari A, Sood N, Vegad MM, Mehta DC. Epidemiological and microbiological profile of infective keratitis in Ahmedabad. Indian J Ophthalmol. 2012;60(4):267-272. doi:10.4103/0301-4738.98702

9. Dhakhwa K, Sharma MK, Bajimaya S, Dwivedi AK, Rai S. Causative organisms in microbial keratitis, their sensitivity pattern and treatment outcome in western Nepal. Nepal $J$ Ophthalmol. 2012;4(1):119-127. doi:10.3126/nepjoph.v4i1.5863

10. Zhang C, Liang Y, Deng S, Wang Z, Li R, Sun X. Distribution of bacterial keratitis and emerging resistance to antibiotics in China from 2001 to 2004. Clin Ophthalmol. 2008;2(3):575-579. doi:10.2147/opth.s 1774

11. Toshida H, Kogure N, Inoue N, Murakami A. Trends in microbial keratitis in Japan. Eye Contact Lens. 2007;33(2):70-73. doi:10.1097/ 01.icl.0000237825.98225.ca

12. Hong J, Xu J, Hua J, Sun X. Bacterial keratitis in Shanghai. Ophthalmology. 2013;120(3):647. doi:10.1016/j.ophtha.2012.10.038

13. Fong CF, Tseng CH, Hu FR, Wang IJ, Chen WL, Hou YC. Clinical characteristics of microbial keratitis in a university hospital in Taiwan. Am J Ophthalmol. 2004;137(2):329-336. doi:10.1016/j.ajo.2003.09.001

14. Lam DS, Houang E, Fan DS, Lyon D, Seal D, Wong E. Incidence and risk factors for microbial keratitis in Hong Kong: comparison with Europe and North America. Eye (Lond). 2002;16(5):608-618. doi:10.1038/sj.eye.6700151 
15. Xie L, Dong X, Shi W. Treatment of fungal keratitis by penetrating keratoplasty. Br J Ophthalmol. 2001;85(9):1070-1074. doi:10.1136/ bjo.85.9.1070

16. Bajracharya L, Gurung R. Outcome of therapeutic penetrating keratoplasty in a tertiary eye care center in Nepal. Clin Ophthalmol. 2015;9:2299-2304. doi:10.2147/OPTH.S92176

17. Ti SE, Scott JA, Janardhanan P, Tan DT. Therapeutic keratoplasty for advanced suppurative keratitis. Am J Ophthalmol. 2007;143(5):755762. doi:10.1016/j.ajo.2007.01.015

18. Chen WL, Wu CY, Hu FR, Wang IJ. Therapeutic penetrating keratoplasty for microbial keratitis in Taiwan from 1987 to 2001. Am J Ophthalmol. 2004;137(4):736-743. doi:10.1016/j.ajo.2003.11.010

19. Yao YF, Zhang YM, Zhou P, Zhang B, Qiu WY, Tseng SC. Therapeutic penetrating keratoplasty in severe fungal keratitis using cryopreserved donor corneas. Br J Ophthalmol. 2003;87(5):543-547. doi:10.1136/bjo.87.5.543

20. Gupta PC, Ram J. Outcome of therapeutic penetrating keratoplasty using glycerol-preserved donor corneas in infectious keratitis. Cornea. 2017;36(4):e12. doi:10.1097/ICO.0000000000001096
21. Thanathanee O, Sripawadkul W, Anutarapongpan O, Luanratanakorn P, Suwan-Apichon O. Outcome of therapeutic penetrating keratoplasty using glycerol-preserved donor corneas in infectious keratitis. Cornea. 2016;35(9):1175-1178. doi:10.1097/ICO.000000000000 0841

22. Rush SW, Rush RB. Outcomes of infectious versus sterile perforated corneal ulcers after therapeutic penetrating keratoplasty in the United States. J Ophthalmol. 2016;2016:6284595.

23. Croghan C, Chou CY, Gajree S, Ramaesh K, Anijeet D. Emergency therapeutic penetrating keratoplasty in a tertiary ophthalmic care facility. Eye (Lond). 2017;32:655-657.

24. Srujana D, Kaur M, Urkude J, Rathi A, Sharma N, Titiyal JS. Longterm functional and anatomic outcomes of repeat graft after optically failed therapeutic keratoplasty. Am J Ophthalmol. 2018;189:166-175. doi:10.1016/j.ajo.2018.03.011

25. Jan RL, Tai MC, Weng SF, Chang C, Wang JJ, Chang YS. Risk of corneal ulcer in patients with end-stage renal disease: a retrospective large-scale cohort study. $\mathrm{Br} J$ Ophthalmol. 2018;102(7):868-872. doi:10.1136/bjophthalmol-2016-310098
Infection and Drug Resistance

\section{Publish your work in this journal}

Infection and Drug Resistance is an international, peer-reviewed openaccess journal that focuses on the optimal treatment of infection (bacterial, fungal and viral) and the development and institution of preventive strategies to minimize the development and spread of resistance. The journal is specifically concerned with the epidemiology of antibiotic resistance and the mechanisms of resistance development and diffusion in both hospitals and the community. The manuscript management system is completely online and includes a very quick and fair peerreview system, which is all easy to use. Visit http://www.dovepress.com/ testimonials.php to read real quotes from published authors. 